\title{
EXTREMES OF MULTIDIMENSIONAL STATIONARY GAUSSIAN RANDOM FIELDS
}

BY

NATALIA SOJA-KUKIEŁA (TORUŃ)

\begin{abstract}
Let $\left\{X(\mathbf{t}): \mathbf{t}=\left(t_{1}, t_{2}, \ldots, t_{d}\right) \in[0, \infty)^{d}\right\}$ be a centered stationary Gaussian field with almost surely continuous sample paths, unit variance and correlation function $r$ satisfying $r(\mathbf{t})<1$ for every $\mathbf{t} \neq \mathbf{0}$ and $r(\mathbf{t})=1-\sum_{i=1}^{d}\left|t_{i}\right|^{\alpha_{i}}+o\left(\sum_{i=1}^{d}\left|t_{i}\right|^{\alpha_{i}}\right)$, as $\mathbf{t} \rightarrow \mathbf{0}$, with some $\alpha_{1}, \alpha_{2}, \ldots, \alpha_{d} \in(0,2]$. The main result of this contribution is the description of the asymptotic behaviour of $\mathrm{P}\left(\sup \left\{X(\mathbf{t}): \mathbf{t} \in \mathcal{J}_{\mathbf{m}}^{\mathbf{x}}\right\} \leqslant u\right)$, as $u \rightarrow \infty$, for some Jordan-measurable sets $\mathcal{J}_{\mathbf{m}}^{\mathbf{x}}$ of volume proportional to $\mathrm{P}\left(\sup \left\{X(\mathbf{t}): \mathbf{t} \in[0,1]^{d}\right\}>u\right)^{-1}(1+o(1))$.
\end{abstract}

2010 AMS Mathematics Subject Classification: Primary: 60G15; Secondary: 60G70, 60G60.

Key words and phrases: Gaussian random field, supremum, limit theorem, asymptotics, Berman condition, strong dependence.

\section{INTRODUCTION}

In extreme value theory of Gaussian processes, we have the following seminal result (see Leadbetter et al. [4], Theorem 12.3.4; Arendarczyk and Dębicki [I]], Lemma 4.3; Tan and Hashorva [7], Lemma 3.3) concerning the asymptotics of the distribution of supremum of a centered stationary Gaussian process $\{X(t): t \geqslant 0\}$ with correlation function satisfying

$$
r(t)=\operatorname{Cov}(X(t), X(0))=1-|t|^{\alpha}+o\left(|t|^{\alpha}\right), \quad \text { as } t \rightarrow 0,
$$

for some $\alpha \in(0,2]$, over intervals with length proportional to

$$
\mu(u)=\mathrm{P}\left(\sup _{t \in[0,1]} X(t)>u\right)^{-1}(1+o(1)), \quad \text { as } u \rightarrow \infty .
$$

THEOREM 1.1. Let $\{X(t): t \geqslant 0\}$ be a zero-mean, unit-variance stationary Gaussian process with a.s. continuous sample paths and correlation function $r$ satisfying $(\mathbb{L} \mathbb{L})$ and $r(t) \log t \rightarrow R \in[0, \infty)$ as $t \rightarrow \infty$. Let $0<A<B<\infty$. 
Then

$$
\mathrm{P}\left(\sup _{t \in[0, x \mu(u)]} X(t) \leqslant u\right) \rightarrow \mathrm{E} \exp (-x \exp (-R+\sqrt{2 R} \mathcal{W})),
$$

as $u \rightarrow \infty$, uniformly for $x \in[A, B]$, with $\mathcal{W}$ an $N(0,1)$ random variable.

It is natural to study a similar problem in the $d$-dimensional setting for arbitrary $d \in \mathbb{N}$. In this case one considers a centered stationary Gaussian process $\left\{X\left(t_{1}, t_{2}, \ldots, t_{d}\right): t_{1}, t_{2}, \ldots, t_{d} \geqslant 0\right\}$ with unit variance and correlation function $r\left(t_{1}, t_{2}, \ldots, t_{d}\right)=\operatorname{Cov}\left(X\left(t_{1}, t_{2}, \ldots, t_{d}\right), X(0,0, \ldots, 0)\right)$ satisfying

$$
r\left(t_{1}, t_{2}, \ldots, t_{d}\right)=1-\sum_{i=1}^{d}\left|t_{i}\right|^{\alpha_{i}}+o\left(\sum_{i=1}^{d}\left|t_{i}\right|^{\alpha_{i}}\right),
$$

as $t_{1}, t_{2}, \ldots, t_{d} \rightarrow 0$, with $\alpha_{1}, \alpha_{2}, \ldots, \alpha_{d} \in(0,2]$. The subject of interest is then the distribution of supremum of the field $\left\{X\left(t_{1}, t_{2}, \ldots, t_{d}\right)\right\}$ over sets of volume proportional to

$$
m(u)=\mathrm{P}\left(\sup _{\left(t_{1}, t_{2}, \ldots, t_{d}\right) \in[0,1]^{d}} X\left(t_{1}, t_{2}, \ldots, t_{d}\right)>u\right)^{-1}(1+o(1)) .
$$

In this paper we investigate suprema over sets of the form

$$
\mathcal{J}_{\mathbf{m}}^{\mathbf{x}}:=\left\{\left(t_{1}, t_{2}, \ldots, t_{d}\right) \in \mathbb{R}^{d}:\left(\frac{t_{1}}{x_{1} m_{1}(u)}, \frac{t_{2}}{x_{2} m_{2}(u)}, \ldots, \frac{t_{d}}{x_{d} m_{d}(u)}\right) \in \mathcal{J}\right\},
$$

where $\mathcal{J} \subset \mathbb{R}^{d}$ is a Jordan-measurable set with Lebesgue measure $\lambda(\mathcal{J})>0$, $\mathbf{x}=\left(x_{1}, x_{2}, \ldots, x_{d}\right) \in(0, \infty)^{d}$ and $\mathbf{m}=\left(m_{1}, m_{2}, \ldots, m_{d}\right)$ with $m_{1}, m_{2}, \ldots, m_{d}$ some positive functions satisfying $m_{1}(u) m_{2}(u) \ldots m_{d}(u)=m(u)$. Let us put $\mathcal{J}_{\mathbf{m}}:=\mathcal{J}_{\mathbf{m}}^{(1, \ldots, 1)}$. One interesting case is $\mathcal{J}=[0,1]^{d}$ with $\mathcal{J}_{\mathbf{m}}^{\mathbf{x}}=\prod_{i=1}^{d}\left[0, x_{i} m_{i}(u)\right]$.

In a recent paper Dębicki et al. [3] consider the case $d=2$. They assume that the functions $m_{1}$ and $m_{2}$ tend to infinity and satisfy

$$
\frac{\log m_{1}(u)}{\log m_{2}(u)} \rightarrow 1 \quad \text { as } u \rightarrow \infty .
$$

The authors establish the following two-dimensional counterpart ([3], Theorem 2) of Theorem ㄸ.].

THEOREM 1.2. Let $\left\{X\left(t_{1}, t_{2}\right): t_{1}, t_{2} \geqslant 0\right\}$ be a zero-mean, unit-variance stationary Gaussian field with a.s. continuous sample paths and correlation function $r$ satisfying ([L.2) and $r\left(t_{1}, t_{2}\right) \log \sqrt{t_{1}^{2}+t_{2}^{2}} \rightarrow R \in[0, \infty)$ as $t_{1}^{2}+t_{2}^{2} \rightarrow \infty$. Let $m_{1}$ and $m_{2}$ be positive functions such that $m_{1}(u) m_{2}(u)=m(u)$ and ([L.3) hold. Then: 
(i) for each $0<A<B<\infty$,

$$
\mathrm{P}\left(\sup _{\left(t_{1}, t_{2}\right) \in\left[0, x_{1} m_{1}\right] \times\left[0, x_{2} m_{2}\right]} X\left(t_{1}, t_{2}\right) \leqslant u\right) \rightarrow \mathrm{E} e^{-x_{1} x_{2} \exp (-2 R+2 \sqrt{R} \mathcal{W})},
$$

as $u \rightarrow \infty$, uniformly for $\left(x_{1}, x_{2}\right) \in[A, B]^{2}$, with $\mathcal{W}$ an $N(0,1)$ random variable;

(ii) for every Jordan-measurable set $\mathcal{J} \subset \mathbb{R}^{2}$ with Lebesgue measure $\lambda(\mathcal{J})>0$,

$$
\mathrm{P}\left(\sup _{\left(t_{1}, t_{2}\right) \in \mathcal{J}_{\mathbf{m}}} X\left(t_{1}, t_{2}\right) \leqslant u\right) \rightarrow \mathrm{E} e^{-\lambda(\mathcal{J}) \exp (-2 R+2 \sqrt{R} \mathcal{W})},
$$

as $u \rightarrow \infty$, with $\mathcal{W}$ an $N(0,1)$ random variable.

Our goal is to derive a general limit theorem for the distribution of supremum of the field $\left\{X\left(t_{1}, t_{2}, \ldots, t_{d}\right)\right\}$ over sets $\mathcal{J}_{\mathbf{m}}^{\mathbf{x}}$, for arbitrary $d \in \mathbb{N}$ and for a wide class of families $\left\{m_{1}, m_{2}, \ldots, m_{d}\right\}$ of functions, uniform for $\mathbf{x} \in[A, B]^{d}$, for all $0<A<B<\infty$. The main result is Theorem B.]. In the paper we do not assume that every $m_{i}$ tends to infinity like Dębicki et al. [3] do. We fully explain the case when all $m_{i}$ s are separated from zero (see Theorem B.D and Remark B.J) and give some partial results in the case when some of $m_{i}$ s tend to zero (see Corollaries B.4 and $(3.5)$.

\section{PRELIMINARIES}

We consider $\mathbb{R}^{d}$ with coordinatewise order $\leqslant$, write $\mathbf{t}=\left(t_{1}, t_{2}, \ldots, t_{d}\right)$ for an element $\mathbf{t} \in \mathbb{R}^{d}$, put $\mathbf{0}:=(0,0, \ldots, 0)$ and $\mathbf{1}:=(1,1, \ldots, 1)$, and denote by $\|\cdot\|_{\infty}$ the sup-norm in $\mathbb{R}^{d}$, i.e., $\|\mathbf{t}\|_{\infty}=\max \left\{\left|t_{1}\right|,\left|t_{2}\right|, \ldots,\left|t_{d}\right|\right\}$ for any $\mathbf{t} \in \mathbb{R}^{d}$.

Let $\left\{X(\mathbf{t}): \mathbf{t} \in[0, \infty)^{d}\right\}$ be a centered stationary Gaussian field with a.s. continuous sample paths, unit variance and correlation function

$$
r(\mathbf{t})=\operatorname{Cov}(X(\mathbf{t}), X(\mathbf{0})) .
$$

We will often assume that the correlation function satisfies:

A1: $r(\mathbf{t})=1-\sum_{i=1}^{d}\left|t_{i}\right|^{\alpha_{i}}+o\left(\sum_{i=1}^{d}\left|t_{i}\right|^{\alpha_{i}}\right)$ as $t_{1}, t_{2}, \ldots, t_{d} \rightarrow 0$;

A2: $r(\mathbf{t})<1$ for $\mathbf{t} \neq \mathbf{0}$;

A3: $r(\mathbf{t}) \log \sqrt{t_{1}^{2}+t_{2}^{2}+\ldots+t_{d}^{2}} \rightarrow R$ as $t_{1}^{2}+t_{2}^{2}+\ldots+t_{d}^{2} \rightarrow \infty$, with some constants $\alpha_{1}, \alpha_{2}, \ldots, \alpha_{d} \in(0,2]$ and $R \in[0, \infty)$.

The above conditions are analogous to the ones given in [4], [I]], [ 7 ], [3].

Condition A1 implies that the correlation function $r$ is continuous. A1 and $\mathbf{A 2}$ give $|r(\mathbf{t})|<1$ for $\mathbf{t} \neq \mathbf{0}$. Moreover, condition A2 follows from A1 and A3. Notice that we study both weakly dependent fields, satisfying $\mathbf{A 3}$ with $R=0$, and strongly dependent fields, satisfying $\mathbf{A} \mathbf{3}$ with $R \in(0, \infty)$.

For every $\alpha \in(0,2]$, we denote by $\mathcal{H}_{\alpha}$ the Pickands constant (see [5]), i.e.,

$$
\mathcal{H}_{\alpha}:=\lim _{T \rightarrow \infty} \frac{\operatorname{E} \exp \left(\max _{0 \leqslant t \leqslant T} B_{\alpha / 2}(t)-|t|^{\alpha}\right)}{T},
$$

where $\left\{B_{\alpha / 2}(t): t \geqslant 0\right\}$ is a fractional Brownian motion with Hurst index $\alpha / 2$. 
Let $\mathcal{W}$ be a standard normal random variable and let $\Phi(u):=\mathrm{P}(\mathcal{W} \leqslant u)$, $\Psi(u):=\mathrm{P}(\mathcal{W}>u)$. We recall that

$$
\Psi(u)=\frac{1}{\sqrt{2 \pi} u} \exp \left(-\frac{u^{2}}{2}\right)(1+o(1)) \quad \text { as } u \rightarrow \infty .
$$

If the considered field $\{X(\mathbf{t})\}$ satisfies $\mathbf{A 1}$ and $\mathbf{A 2}$, then, for arbitrary Jordanmeasurable set $\mathcal{J} \subset \mathbb{R}^{d}$ with Lebesgue measure $\lambda(\mathcal{J})>0$, we have

$$
\mathrm{P}\left(\max _{\mathbf{t} \in \mathcal{J}} X(\mathbf{t})>u\right)=\lambda(\mathcal{J}) \prod_{i=1}^{d}\left(\mathcal{H}_{\alpha_{i}} u^{2 / \alpha_{i}}\right) \Psi(u)(1+o(1)),
$$

as $u \rightarrow \infty$, due to Piterbarg [6], Theorem 7.1. Thus

$$
m(u):=\left(\prod_{i=1}^{d}\left(\mathcal{H}_{\alpha_{i}} u^{2 / \alpha_{i}}\right) \Psi(u)\right)^{-1}=\mathrm{P}\left(\max _{\mathbf{t} \in[0,1]^{d}} X(\mathbf{t})>u\right)^{-1}(1+o(1)) .
$$

Let $m_{1}, m_{2}, \ldots, m_{d}$ be positive functions such that

$$
m_{1}(u) m_{2}(u) \ldots m_{d}(u)=m(u)
$$

and for some $k \in\{0,1, \ldots, d-1\}$ :

1. for every $i \in\{1,2, \ldots, k\}$ there exists an $M_{i} \in(0, \infty)$ such that

$$
m_{i}(u) \rightarrow M_{i} \quad \text { as } u \rightarrow \infty
$$

2. for every $i \in\{k+1, k+2, \ldots, d\}$ we have

$$
m_{i}(u) \rightarrow \infty(\text { as } u \rightarrow \infty) \quad \text { and } \quad m_{i}(u)=\exp \left(\gamma_{i} u^{2}\right) c_{i}(u),
$$

for some constant $\gamma_{i} \in[0,1 / 2]$ and positive function $c_{i}$ with $\log c_{i}(u)=o\left(u^{2}\right)$. Then $\gamma_{k+1}+\gamma_{k+2}+\ldots+\gamma_{d}=1 / 2$. We put $\gamma:=\max _{i} \gamma_{i}$.

For arbitrary $\mathbf{x} \in(0, \infty)^{d}$, we define $\mathcal{R}^{\mathbf{x}}:=\left[0, x_{1}\right] \times\left[0, x_{2}\right] \times \ldots \times\left[0, x_{d}\right]$ and $\mathcal{R}_{\mathbf{m}}^{\mathbf{x}}:=\left[0, x_{1} m_{1}(u)\right] \times\left[0, x_{2} m_{2}(u)\right] \times \ldots \times\left[0, x_{d} m_{d}(u)\right]$ for each $u \in \mathbb{R}$. Note that $\mathcal{R}_{\mathbf{m}}^{\mathbf{x}}=\mathcal{J}_{\mathbf{m}}^{\mathbf{x}}$ for $\mathcal{J}=[0,1]^{d}$.

\section{RESULTS}

Below, in Section B.1, we present Theorem B.1, which is the main result. Its proof is given in Sections 3.3 and 13.4. Some consequences of Theorem B.] can be found in Sections 3.1 and 3.2 . 
3.1. Main theorem. The following theorem describes the asymptotic behaviour of $\mathrm{P}\left(\sup \left\{X(\mathbf{t}): \mathbf{t} \in \mathcal{J}_{\mathbf{m}}^{\mathbf{x}}\right\} \leqslant u\right)$, as $u \rightarrow \infty$, for Jordan-measurable sets $\mathcal{J}_{\mathbf{m}}^{\mathbf{x}}$ of volume proportional to $m(u)$.

THEOREM 3.1. Let $\left\{X(\mathbf{t}): \mathbf{t} \in[0, \infty)^{d}\right\}$ be a centered stationary Gaussian field with a.s. continuous sample paths, unit variance and correlation function $r$ that satisfies $\mathbf{A} 1$ and $\mathbf{A 3}$ with some $R \in[0, \infty)$. Then, for every Jordan-measurable set $\mathcal{J} \subset \mathbb{R}^{d}$ with $\lambda(\mathcal{J})>0$, for each $0<A<B<\infty$,

$$
\mathrm{P}\left(\sup _{\mathbf{t} \in \mathcal{J}_{\mathbf{m}}^{\mathbf{x}}} X(\mathbf{t}) \leqslant u\right) \rightarrow \mathrm{E} \exp \left(-x_{1} x_{2} \ldots x_{d} \lambda(\mathcal{J}) \exp \left(-\frac{R}{2 \gamma}+\sqrt{\frac{R}{\gamma}} \mathcal{W}\right)\right)
$$

as $u \rightarrow \infty$, uniformly for $\mathrm{x} \in[A, B]^{d}$.

Applying the above theorem for $\mathcal{J}=[0,1]^{d}$, we obtain the following result.

COROLLARY 3.1. Let $\{X(\mathbf{t})\}$ satisfy the assumptions of Theorem $\mathbf{B}$.]. Then, for each $0<A<B<\infty$,

$$
\mathrm{P}\left(\sup _{\mathbf{t} \in \mathcal{R}_{\mathbf{m}}^{\mathbf{x}}} X(\mathbf{t}) \leqslant u\right) \rightarrow \mathrm{E} \exp \left(-x_{1} x_{2} \ldots x_{d} \exp \left(-\frac{R}{2 \gamma}+\sqrt{\frac{R}{\gamma}} \mathcal{W}\right)\right),
$$

as $u \rightarrow \infty$, uniformly for $\mathrm{x} \in[A, B]^{d}$.

In the special case, when $k=0$ and the functions $m_{1}, m_{2}, \ldots, m_{d}$ are chosen so that $\gamma_{1}=\gamma_{2}=\ldots=\gamma_{d}$ (and thus a $d$-dimensional analog of ([1.3) holds), we have the following corollary. Note that for $d=2$ it coincides with Theorem L.2.

COROLLARY 3.2. Let the assumptions of Theorem 3.1 be satisfied and let

$$
\frac{\log m_{i}(u)}{\log m_{j}(u)} \rightarrow 1 \text { as } u \rightarrow \infty, \quad \text { for } i, j \in\{1,2, \ldots, d\} .
$$

Then, for every Jordan-measurable set $\mathcal{J} \subset \mathbb{R}^{d}$ with $\lambda(\mathcal{J})>0$,

$$
\mathrm{P}\left(\sup _{\mathbf{t} \in \mathcal{J}_{\mathbf{m}}^{\mathbf{x}}} X(\mathbf{t}) \leqslant u\right) \rightarrow \mathrm{E} \exp \left(-x_{1} x_{2} \ldots x_{d} \lambda(\mathcal{J}) \exp (-d R+\sqrt{2 d R} \mathcal{W})\right),
$$

as $u \rightarrow \infty$, uniformly for $\mathrm{x} \in[A, B]^{d}$, for each $0<A<B<\infty$.

3.2. Some consequences of the main theorem. Let the field $\{X(\mathbf{t})\}$ satisfy the assumptions of Theorem 3. . In this section we ask for the asymptotic behaviour of the supremum of $\{X(\mathbf{t})\}$ over sets $\mathcal{J}_{\mathbf{m}}^{\mathbf{x}}$, for $\mathcal{J} \subset \mathbb{R}^{d}$ a Jordan-measurable set with $\lambda(\mathcal{J})>0, \mathbf{x} \in(0, \infty)^{d}, \overline{\mathbf{m}}=\left(\bar{m}_{1}, \bar{m}_{2}, \ldots, \bar{m}_{d}\right)$ and $\bar{m}_{1}, \bar{m}_{2}, \ldots, \bar{m}_{d}$ some positive functions with $\bar{m}_{1}(u) \bar{m}_{2}(u) \ldots \bar{m}_{d}(u)=m(u)$. Note, we do not assume that $\bar{m}_{1}, \bar{m}_{2}, \ldots, \bar{m}_{d}$ fulfill all the conditions, which have to be satisfied by the functions $m_{1}, m_{2}, \ldots, m_{d}$ introduced in Section $\square$. 
First, we consider the case when the functions $\bar{m}_{1}, \bar{m}_{2}, \ldots, \bar{m}_{d}$ are separated from zero, i.e., $\bar{m}_{1}(u), \bar{m}_{2}(u), \ldots, \bar{m}_{d}(u)>\varepsilon$ for some $\varepsilon>0$. Then, it is easy to show that every sequence $\left\{u_{n}\right\}_{n \in \mathbb{N}}$ tending to infinity contains a subsequence $\left\{u_{n_{j}}\right\}_{j \in \mathbb{N}}$ such that for each $i \in\{1,2, \ldots, d\}$ we have $\bar{m}_{i}\left(u_{n_{j}}\right) \rightarrow \bar{M}_{i} \in[\varepsilon, \infty)$, as $j \rightarrow \infty$, or, alternatively, $\bar{m}_{i}\left(u_{n_{j}}\right)=\exp \left(\bar{\gamma}_{i} u_{n_{j}}^{2}\right) \bar{c}_{i}\left(u_{n_{j}}\right) \rightarrow \infty$, as $j \rightarrow \infty$, for some constant $\bar{\gamma}_{i} \in[0,1 / 2]$ and some function $\bar{c}_{i}$ with $\log \bar{c}_{i}\left(u_{n_{j}}\right)=o\left(u_{n_{j}}^{2}\right)$. We can apply Theorem 3.0 for such subsequences. This justifies the following remark.

REMARK 3.1. Theorem B.] fully explains the case when $\bar{m}_{1}, \bar{m}_{2}, \ldots, \bar{m}_{d}$ are positive functions separated from zero, such that $\bar{m}_{1}(u) \bar{m}_{2}(u) \ldots \bar{m}_{d}(u)=m(u)$. It gives the asymptotics for convergent subsequences.

Since for weakly dependent Gaussian fields the limit in Theorem B.] does not depend on $\gamma$, the above considerations entail a concise corollary.

COROLLARY 3.3. Let $\{X(\mathbf{t})\}$ satisfy the assumptions of Theorem 3.$]$ with $R=0$ and let $\bar{m}_{1}, \bar{m}_{2}, \ldots, \bar{m}_{d}$ be positive functions separated from zero, such that $\bar{m}_{1}(u) \bar{m}_{2}(u) \ldots \bar{m}_{d}(u)=m(u)$. Then, for each $0<A<B<\infty$,

$$
\mathrm{P}\left(\sup _{\mathbf{t} \in \mathcal{J}_{\mathbf{m}}^{\mathbf{x}}} X(\mathbf{t}) \leqslant u\right) \rightarrow \exp \left(-x_{1} x_{2} \ldots x_{d} \lambda(\mathcal{J})\right),
$$

as $u \rightarrow \infty$, uniformly for $\mathbf{x} \in[A, B]^{d}$.

Next, we focus on the case when $\bar{m}_{i}$ s are allowed to tend to zero. In general, such weakening of the assumptions enforces a different approach. However, basing on Theorem B.], we can give the limit theorems in two special opposite cases: when $\bar{m}_{i} \rightarrow 0$ sufficiently fast and when $\bar{m}_{i} \rightarrow 0$ sufficiently slow.

Suppose that for some $0 \leqslant j \leqslant k<d$ :

0 . for every $i \in\{1,2, \ldots, j\}$ we have

$$
\bar{m}_{i}(u) \rightarrow 0 \quad \text { as } u \rightarrow \infty
$$

1. for every $i \in\{j+1, j+2, \ldots, k\}$ there exists an $\bar{M}_{i} \in(0, \infty)$ such that

$$
\bar{m}_{i}(u) \rightarrow \bar{M}_{i} \quad \text { as } u \rightarrow \infty ;
$$

2. for every $i \in\{k+1, k+2, \ldots, d\}$

$$
\bar{m}_{i}(u) \rightarrow \infty(\text { as } u \rightarrow \infty) \quad \text { and } \quad \bar{m}_{i}(u)=\exp \left(\bar{\gamma}_{i} u^{2}\right) \bar{c}_{i}(u)
$$

hold for some constant $\bar{\gamma}_{i} \geqslant 0$ and function $\bar{c}_{i}$ such that $\log \bar{c}_{i}(u)=o\left(u^{2}\right)$. Then $\bar{\gamma}_{k+1}+\bar{\gamma}_{k+2}+\ldots+\bar{\gamma}_{d} \geqslant 1 / 2$. We put $\bar{\gamma}:=\max _{i} \bar{\gamma}_{i}$.

Note that the above conditions are very similar to the conditions given in Section $\square$ for the functions $m_{1}, m_{2}, \ldots, m_{d}$. Under these assumptions (and some extra ones) we can prove the following results. 
COROLLARY 3.4. Assume that $\bar{m}_{1}, \bar{m}_{2}, \ldots, \bar{m}_{d}$ satisfy the above conditions and, moreover,

$$
\bar{m}_{1}(u)=\exp \left(-\kappa u^{2}\right) c(u)
$$

for some constant $\kappa>0$ and function $c$ satisfying $\log c(u)=o\left(u^{2}\right)$. Then,

$$
\mathrm{P}\left(\sup _{\mathbf{t} \in \mathcal{J}_{\mathbf{m}}^{\mathrm{x}}} X(\mathbf{t}) \leqslant u\right) \rightarrow 0, \quad \text { as } u \rightarrow \infty,
$$

uniformly for $\mathrm{x} \in[A, \infty)^{d}$, for each $A>0$.

P r o o f. Let $\mathbf{x} \in(0, \infty)^{d}$. Since the set $\mathcal{J} \subset \mathbb{R}^{d}$ is Jordan-measurable and $\lambda(\mathcal{J})>0$, there exist $\mathbf{y} \in \mathbb{R}^{d}$ and $\mathbf{z} \in(0, \infty)^{d}$ such that $\mathbf{y}+\mathcal{R}^{\mathbf{z}} \subset \mathcal{J}$. Thus

$$
\mathrm{P}\left(\sup _{\mathbf{t} \in \mathcal{J}_{\mathbf{m}}^{\mathbf{x}}} X(\mathbf{t}) \leqslant u\right) \leqslant \mathrm{P}\left(\sup _{\mathbf{t} \in\left(\mathbf{y}+\mathcal{R}^{\mathbf{z}}\right)_{\mathbf{m}}^{\mathbf{x}}} X(\mathbf{t}) \leqslant u\right)=\mathrm{P}\left(\sup _{\mathbf{t} \in \mathcal{R}_{\mathbf{m}}^{\mathbf{z x}}} X(\mathbf{t}) \leqslant u\right),
$$

with $\mathbf{z x}:=\left(z_{1} x_{1}, z_{2} x_{2}, \ldots, z_{d} x_{d}\right)$, where the last equality is a consequence of stationarity. Furthermore,

$$
\mathrm{P}\left(\sup _{\mathbf{t} \in \mathcal{R}_{\mathbf{m}}^{\mathbf{z x}}} X(\mathbf{t}) \leqslant u\right) \leqslant \mathrm{P}\left(\sup _{0 \leqslant t_{i} \leqslant z_{i} x_{i} \bar{m}_{i}} X\left(0, \ldots, 0, t_{k+1}, t_{k+2}, \ldots, t_{d}\right) \leqslant u\right) .
$$

We will show that the right-hand side of the above inequality tends to zero, using Theorem 3 .] for the field $\hat{X}\left(t_{k+1}, t_{k+2}, \ldots, t_{d}\right):=X\left(0, \ldots, 0, t_{k+1}, t_{k+2}, \ldots, t_{d}\right)$, $t_{k+1}, t_{k+2}, \ldots, t_{d} \geqslant 0$, that satisfies $(d-k)$-dimensional conditions $\mathbf{A 1}$ and $\mathbf{A 3}$.

Since $\kappa>0$, we have $\sigma:=\bar{\gamma}_{k+1}+\bar{\gamma}_{k+2}+\ldots+\bar{\gamma}_{d}>1 / 2$. Hence

$$
\frac{\bar{m}_{k+1}(u) \bar{m}_{k+2}(u) \ldots \bar{m}_{d}(u)}{\hat{m}(u)} \rightarrow \infty, \quad \text { as } u \rightarrow \infty,
$$

where

$$
\hat{m}(u):=\left(\prod_{i=k+1}^{d}\left(\mathcal{H}_{\alpha_{i}} u^{2 / \alpha_{i}}\right) \Psi(u)\right)^{-1} .
$$

For every $i \in\{k+1, k+2, \ldots, d\}$, we put

$$
\hat{m}_{i}(u):=\exp \left(\hat{\gamma}_{i} u^{2}\right) \hat{c}_{i}(u)
$$

with $\hat{\gamma}_{i}:=(2 \sigma)^{-1} \bar{\gamma}_{i}$ and $\hat{c}_{i}(u):=\left(\hat{m}(u) \exp \left(-u^{2} / 2\right)\right)^{1 /(d-k)}$. Then $\hat{\gamma}_{i} \in[0,1 / 2]$, $\log \hat{c}_{i}(u)=o\left(u^{2}\right)$ and $\hat{\gamma}_{k+1}+\hat{\gamma}_{k+2}+\ldots+\hat{\gamma}_{d}=1 / 2$. Moreover, the functions $\hat{m}_{i}$ satisfy $\hat{m}_{k+1}(u) \hat{m}_{k+2}(u) \ldots \hat{m}_{d}(u)=\hat{m}(u)$ and we have

$$
\frac{\bar{m}_{i}(u)}{\hat{m}_{i}(u)} \rightarrow \infty \quad \text { as } u \rightarrow \infty .
$$


Let $C>0$ be arbitrary. Since $\tilde{m}_{i}(u) / \hat{m}_{i}(u)>C$ for all sufficiently large $u$, we obtain

$$
\begin{aligned}
\limsup _{u \rightarrow \infty} \mathrm{P} & \left(\sup _{0 \leqslant t_{i} \leqslant x_{i} \tilde{m}_{i}} X\left(0, \ldots, 0, t_{k+1}, t_{k+2}, \ldots, t_{d}\right) \leqslant u\right) \\
& =\limsup _{u \rightarrow \infty} \mathrm{P}\left(\sup _{0 \leqslant t_{i} \leqslant x_{i} \tilde{m}_{i}} \hat{X}\left(t_{k+1}, t_{k+2}, \ldots, t_{d}\right) \leqslant u\right) \\
& \leqslant \limsup _{u \rightarrow \infty} \mathrm{P}\left(\sup _{0 \leqslant t_{i} \leqslant C x_{i} \hat{m}_{i}} \hat{X}\left(t_{k+1}, t_{k+2}, \ldots, t_{d}\right) \leqslant u\right) \\
& =\operatorname{E} \exp \left(-C^{d-k} x_{k+1} x_{k+2} \ldots x_{d} \exp \left(-\frac{R}{2 \hat{\gamma}}+\sqrt{\left.\left.\frac{R}{\hat{\gamma}} \mathcal{W}\right)\right),}\right.\right.
\end{aligned}
$$

with $\hat{\gamma}:=\max _{i} \hat{\gamma}_{i}$, due to Theorem B.1. Since the right-hand side tends to zero as $C \rightarrow \infty$, the proof of pointwise convergence is complete. Uniform convergence simply follows from the monoticity of $\mathbf{x} \mapsto \mathrm{P}\left(\sup \left\{X(\mathbf{t}) \leqslant u: \mathbf{t} \in \mathcal{J}_{\overline{\mathbf{m}}}^{\mathbf{x}}\right\}\right)$.

COROLLARY 3.5. Suppose that $m_{1}, m_{2}, \ldots, m_{d}$ are positive functions such that $m_{1}(u) m_{2}(u) \ldots m_{d}(u)=m(u)$ holds and, moreover, assume that

$$
\begin{gathered}
m_{i}(u)=1, \quad \text { for } i \leqslant j, \text { for some } j \in\{1,2, \ldots, d-1\}, \\
m_{i}(u)=\exp \left(\gamma_{i} u^{2}\right) c_{i}(u) \rightarrow M_{i} \in(0, \infty] \text {, as } u \rightarrow \infty, \quad \text { for } i>j,
\end{gathered}
$$

where $\gamma_{i} \in[0,1 / 2]$ and $\log c_{i}(u)=o\left(u^{2}\right)$. There exist some positive functions $\nu_{1}, \nu_{2}, \ldots, \nu_{j}$ satisfying $\nu_{i}(u) \rightarrow 0$, such that for all $\bar{m}_{1}, \bar{m}_{2}, \ldots, \bar{m}_{d}$ satisfying the conditions: $\nu_{i}(u)=o\left(\bar{m}_{i}(u)\right)$ for each $i \in\{1,2, \ldots, j\}, \bar{m}_{i}(u)=m_{i}(u)$ for each $i \in\{j+1, j+2, \ldots, d-1\}$ and $\bar{m}_{d}(u)=m_{d}(u) \cdot \prod_{i=1}^{j} \bar{m}_{i}(u)^{-1}$, we have

$$
\mathrm{P}\left(\sup _{\mathbf{t} \in \mathcal{J}_{\overline{\mathbf{m}}}} X(\mathbf{t}) \leqslant u\right) \rightarrow \operatorname{E} \exp \left(-\lambda(\mathcal{J}) \exp \left(-\frac{R}{2 \gamma}+\sqrt{\frac{R}{\gamma}} \mathcal{W}\right)\right)
$$

as $u \rightarrow \infty$.

Proof. Let $\varepsilon_{1}, \varepsilon_{2}, \ldots, \varepsilon_{j}>0$ and $\varepsilon:=\left(\varepsilon_{1}, \varepsilon_{2}, \ldots, \varepsilon_{j}, 1, \ldots, 1, \prod_{i=1}^{j} \varepsilon_{i}^{-1}\right)$. By application of Theorem B.1, we obtain

$$
\mathrm{P}\left(\sup _{\mathbf{t} \in \mathcal{J}_{\mathbf{m}}^{\varepsilon}} X(\mathbf{t}) \leqslant u\right) \rightarrow \operatorname{E} \exp \left(-\lambda(\mathcal{J}) \exp \left(-\frac{R}{2 \gamma}+\sqrt{\frac{R}{\gamma}} \mathcal{W}\right)\right)
$$

as $u \rightarrow \infty$, uniformly for $\varepsilon \in[A, B]^{d}$, for all $0<A<B<\infty$. Note that the above limit does not depend on the choice of $\varepsilon_{1}, \varepsilon_{2}, \ldots, \varepsilon_{j}$. It is not difficult to show that there exist some functions $\nu_{i}, i \in\{1,2, \ldots, j\}$, tending to zero, such 
that for positive functions $\varepsilon_{i}=\varepsilon_{i}(u), i \in\{1,2, \ldots, j\}$, tending to zero, and for $\varepsilon(u):=\left(\varepsilon_{1}(u), \varepsilon_{2}(u), \ldots, \varepsilon_{j}(u), 1, \ldots, 1, \prod_{i=1}^{j} \varepsilon_{i}^{-1}(u)\right)$, we have

$$
\mathrm{P}\left(\sup _{\mathbf{t} \in \mathcal{J}_{\mathbf{m}}^{\boldsymbol{\varepsilon}(u)}} X(\mathbf{t}) \leqslant u\right) \rightarrow \mathrm{E} \exp \left(-\lambda(\mathcal{J}) \exp \left(-\frac{R}{2 \gamma}+\sqrt{\frac{R}{\gamma}} \mathcal{W}\right)\right),
$$

whenever $\nu_{i}(u)=o\left(\varepsilon_{i}(u)\right)$. We shall put $\varepsilon_{i}(u)=\bar{m}_{i}(u)$ for $i \in\{1,2, \ldots, j\}$.

REMARK 3.2. We do not know the form of the functions $\nu_{1}, \nu_{2}, \ldots, \nu_{j}$ from Corollary 3.5. Our conjecture is that $\nu_{i}(u)=u^{-2 / \alpha_{i}}$ for $i \in\{1,2, \ldots, j\}$.

3.3. Lemmas. The lemmas formulated in this section are crucial in the proof of Theorem B.] (see Section 13.4). They are $d$-dimensional counterparts of known results: Lemma B.] generalizes Lemma 12.2.11 in [4] and Lemma 1 in [3]; Lemma 3.3 combines $d$-dimensional analogs of Lemma 12.3.1 in [4] (for weakly dependent fields) and Lemma 3.1 in [⿴囗十 (for strongly dependent fields), it is a generalization of Lemma 2 in [3]. Since the argumentation for Lemmas B.] and 3.2 mimics the one given in [4] and expanded in [团, [2], the proofs are skipped. We present the proof of Lemma 3.3, which improves the lemma given by Dębicki et al. [3], [2] and enables us to establish far more general results than the ones in [3].

Let $a>0$. Put $q_{i}=q_{i}(u):=a u^{-2 / \alpha_{i}}$ for $i \in\{1,2, \ldots, d\}$. Moreover, define $\mathbf{j q}=\mathbf{j q}(u):=\left(j_{1} q_{1}(u), j_{2} q_{2}(u), \ldots, j_{d} q_{d}(u)\right)$ for $\mathbf{j}=\left(j_{1}, j_{2}, \ldots, j_{d}\right) \in \mathbb{Z}^{d}$.

LEMma 3.1. Assume that conditions $\mathbf{A 1}$ and $\mathbf{A 2}$ hold. Then there exists a function $\vartheta$ satisfying $\vartheta(a) \rightarrow 0$, as $a \rightarrow 0$, such that for every $a>0$ we have

$$
\mathrm{P}\left(\sup _{\mathbf{j q} \in \mathbf{y}+\mathcal{R}^{\mathbf{x}}} X(\mathbf{j q}) \leqslant u\right)-\mathrm{P}\left(\sup _{\mathbf{t} \in \mathbf{y}+\mathcal{R}^{\mathbf{x}}} X(\mathbf{t}) \leqslant u\right) \leqslant \frac{x_{1} x_{2} \ldots x_{d}}{m} \vartheta(a)+o\left(\frac{1}{m}\right),
$$

as $u \rightarrow \infty$, uniformly for $\mathbf{y} \in[0, \infty)^{d}$ and $\mathbf{x} \in[A, B]^{d}$, for all $0<A<B<\infty$.

REMARK 3.3. An explicit formula for $\vartheta$ from Lemma [.] can be found in [2].

Lemma 3.2. Suppose that $T=T(u) \rightarrow \infty$ as $u \rightarrow \infty$. Then, providing that conditions $\mathbf{A 1}$ and $\mathbf{A 2}$ are fulfilled, there exists an $\varepsilon>0$ such that for all $R \geqslant 0$

$$
\begin{array}{r}
\frac{m}{q_{1} q_{2} \ldots q_{d}} \sum_{\substack{\mathbf{j} \mathbf{j} \in(-\varepsilon, \varepsilon) \\
\mathbf{j} \neq(0,0, \ldots, 0)}}\left[(1-r(\mathbf{j q})) \frac{R}{\log T}\left(1-\left(r(\mathbf{j q})+(1-r(\mathbf{j q})) \frac{R}{\log T}\right)^{2}\right)^{-1 / 2}\right. \\
\left.\quad \times \exp \left(-\frac{u^{2}}{1+r(\mathbf{j q})+(1-r(\mathbf{j q})) R / \log T}\right)\right] \rightarrow 0,
\end{array}
$$

as $u \rightarrow \infty$. 
Let $R \geqslant 0$ be fixed. The last lemma concerns functions $\rho_{T}$ and $\varrho_{T}$ defined for an arbitrary $T>1$ and for $\mathbf{t} \in \mathbb{R}^{d}$ as follows:

$$
\begin{gathered}
\rho_{T}(\mathbf{t}):= \begin{cases}1, & \max \left\{\left|t_{k+1}\right|,\left|t_{k+2}\right|, \ldots,\left|t_{d}\right|\right\}<1, \\
\left|r(\mathbf{t})-\frac{R}{\log T}\right|, & \text { otherwise; }\end{cases} \\
\varrho_{T}(\mathbf{t}):= \begin{cases}|r(\mathbf{t})|+(1-r(\mathbf{t})) \frac{R}{\log T}, & \max \left\{\left|t_{k+1}\right|,\left|t_{k+2}\right|, \ldots,\left|t_{d}\right|\right\}<1, \\
\frac{R}{\log T}, & \text { otherwise. }\end{cases}
\end{gathered}
$$

Lemma 3.3. Assume that $T_{i}=T_{i}(u) \sim \tau_{i} m_{i}(u)$, as $u \rightarrow \infty$, for some $\tau_{i}>0$ and every $i \in\{1,2, \ldots, d\}$. Let $\varepsilon>0$. Then, providing that conditions $\mathbf{A} 1$ and $\mathbf{A 3}$ with $R \in[0, \infty)$ are fulfilled,

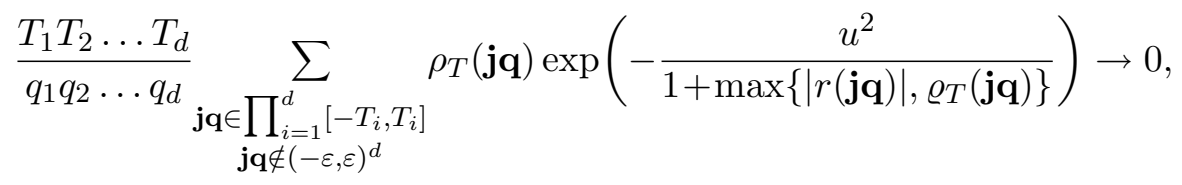

as $u \rightarrow \infty$, with $T:=\max \left\{T_{1}, T_{2}, \ldots, T_{d}\right\}$.

Proof. We present the proof in the case $d=2$. The argumentation for other dimensions is analogous. We follow the reasoning from Lemma 2 in [2], making modifications and skipping some details, which can be found in [2].

Since $T_{1}(u) T_{2}(u) \sim \tau_{1} \tau_{2} m(u)$, as $u \rightarrow \infty$, we get

$$
u^{2}=2 \log \left(T_{1} T_{2}\right)+\left(\frac{2}{\alpha_{1}}+\frac{2}{\alpha_{2}}-1\right) \log \log \left(T_{1} T_{2}\right)+O(1) .
$$

It is not difficult to see that there exists a constant $\delta \in(0,1)$ such that for all sufficiently large $L$

$$
\sup _{\varepsilon \leqslant\|\mathbf{t}\|_{\infty} \leqslant L} \max \left\{|r(\mathbf{t})|, \varrho_{L}(\mathbf{t})\right\}<\delta
$$

Denote by $\beta$ a constant satisfying $0<\beta<(1-\delta) /(1+\delta)$ and divide the set $\mathcal{Q}:=\left[-T_{1}, T_{1}\right] \times\left[-T_{2}, T_{2}\right]-(-\varepsilon, \varepsilon)^{2}$ into two subsets:

$$
\begin{aligned}
\mathcal{S}^{*} & :=\left\{\mathbf{t} \in \mathcal{Q}:\left|t_{1}\right| \leqslant m(u)^{\beta / 2},\left|t_{2}\right| \leqslant m(u)^{\beta / 2}\right\}, \\
\mathcal{S} & :=\mathcal{Q}-\mathcal{S}^{*} .
\end{aligned}
$$

Observe that the shape of the set $\mathcal{S}^{*}$ of volume $m(u)^{\beta}(1+o(1))$ does not depend on the choice of $m_{1}$ and $m_{2}$. 
Following line-by-line the arguments from [2], thanks to the proper choice of $\beta$, we obtain

$$
\frac{T_{1} T_{2}}{q_{1} q_{2}} \sum_{\mathbf{j} \mathbf{q} \in \mathcal{S}^{*}} \rho_{T}(\mathbf{j q}) \exp \left(-\frac{u^{2}}{1+\max \left\{|r(\mathbf{j q})|, \varrho_{T}(\mathbf{j q})\right\}}\right) \rightarrow 0
$$

as $u \rightarrow \infty$.

To complete the proof, it suffices to show that

$$
\frac{T_{1} T_{2}}{q_{1} q_{2}} \sum_{\mathbf{j q} \in \mathcal{S}} \rho_{T}(\mathbf{j q}) \exp \left(-\frac{u^{2}}{1+\max \left\{|r(\mathbf{j q})|, \varrho_{T}(\mathbf{j q})\right\}}\right) \rightarrow 0,
$$

as $u \rightarrow \infty$. By an argument from [2] and the fact that $m(u)^{\beta / 2} \rightarrow \infty$, we get

$$
\max \left\{|r(\mathbf{j q})|, \varrho_{T}(\mathbf{j q})\right\} \leqslant \frac{C}{\log m(u)^{\beta / 2}},
$$

for sufficiently large $u$, some constant $C>0$ and all points jq $\in \mathcal{Q}$ satisfying $\|\mathbf{j q}\|_{\infty} \geqslant m(u)^{\beta / 2}$. Hence we have

$$
\begin{aligned}
& \frac{T_{1} T_{2}}{q_{1} q_{2}} \sum_{\mathbf{j q} \in \mathcal{S}} \rho_{T}(\mathbf{j q}) \exp \left(-\frac{u^{2}}{1+\max \left\{|r(\mathbf{j q})|, \varrho_{T}(\mathbf{j q})\right\}}\right) \\
& \leqslant 4 \frac{T_{1}^{2} T_{2}^{2}}{q_{1}^{2} q_{2}^{2}} \exp \left(-u^{2}\left(1-\frac{C}{\log m^{\beta / 2}}\right)\right) \frac{1}{\log m^{\beta / 2}} \\
& \quad \times \frac{q_{1} q_{2} \log m^{\beta / 2}}{T_{1} T_{2}} \sum_{\mathbf{j} \mathbf{q} \in \mathcal{S}}\left|r(\mathbf{j q})-\frac{R}{\log T}\right| \\
&=: I_{1}(u) \times I_{2}(u) .
\end{aligned}
$$

Applying the equality (B.3) $)$, the definition of the functions $q_{1}$ and $q_{2}$ and the convergence $\log \left(T_{1}(u) T_{2}(u)\right) / \log m(u)^{\beta / 2} \rightarrow 2 / \beta$, as $u \rightarrow \infty$, we conclude that $I_{1}$ is bounded. Our argumentation is analogous to the one given in [2]. The strong condition (ㅍ.3) turns out not to be necessary.

In the next step we prove that $I_{2}(u) \rightarrow 0$ as $u \rightarrow \infty$. Observe that we have

$$
\begin{aligned}
I_{2}(u) \leqslant & \frac{q_{1} q_{2}}{T_{1} T_{2}} \sum_{\mathbf{j} \mathbf{q} \in \mathcal{S}}\left|r(\mathbf{j q}) \log \sqrt{\left(j_{1} q_{1}\right)^{2}+\left(j_{2} q_{2}\right)^{2}}-R\right|(1+o(1)) \\
& +\beta R \frac{q_{1} q_{2}}{T_{1} T_{2}} \sum_{\mathbf{j q} \in \mathcal{S}}\left|1-\frac{\log T}{\log \sqrt{\left(j_{1} q_{1}\right)^{2}+\left(j_{2} q_{2}\right)^{2}}}\right|(1+o(1)) \\
= & : J_{1}(u)+J_{2}(u) .
\end{aligned}
$$


We need to show that both $J_{1}$ and $J_{2}$ tend to zero. Note that $J_{1}(u) \rightarrow 0$ as $u \rightarrow \infty$, due to A3. Additionally,

$$
J_{2}(u) \leqslant \frac{2 R}{\log m} \frac{q_{1} q_{2}}{T_{1} T_{2}} \sum_{\mathbf{j q} \in \mathcal{S}}\left|\log \left(\frac{\sqrt{\left(j_{1} q_{1}\right)^{2}+\left(j_{2} q_{2}\right)^{2}}}{T}\right)\right|,
$$

and hence

$$
J_{2}(u)=\frac{2 R}{\log m} \cdot O\left(\int_{0}^{1} \int_{0}^{1}\left|\log \left(\sqrt{x^{2}+y^{2}}\right)\right| d x d y+\int_{0}^{1}|\log | x|| d x\right) .
$$

Thus (B.5) holds. The combination of (B.4) and (3.5) completes the proof.

3.4. Proof of Theorem B.1. To establish the main result, we develop the ideas given in [4], [U], [ [7] , [3]. The following proof of Theorem B.] combines the method of proof of Theorem L.2 for $d=2$ and $\gamma_{1}=\gamma_{2}=1 / 4$ (see [3], Theorem 2), the lemmas from Section 3.3 and some new observations.

The proof consists of two parts. In (i), we present a complete argumentation for the special case $\mathcal{J}=[0,1]^{d}$. In (ii), we explain how to apply the first part of the proof to obtain the limit theorem for arbitrary $\mathcal{J}$.

(i) Let us consider $\mathcal{J}=[0,1]^{d}$. Then $\mathcal{J}_{\mathbf{m}}^{\mathbf{x}}=\mathcal{R}_{\mathbf{m}}^{\mathbf{x}}$ for $\mathbf{x} \in(0, \infty)^{d}$. Let $\left\{X^{\mathbf{k}}(\mathbf{t})\right\}$, for $\mathbf{k} \in \mathbb{N}^{d-k}$, be independent copies of $\{X(\mathbf{t})\}$ and let

$$
\eta(\mathbf{t}):=X^{\mathbf{k}(\mathbf{t})}(\mathbf{t}) \quad \text { for } \mathbf{t} \in[0, \infty)^{d},
$$

with $\mathbf{k}(\mathbf{t})=\left(\left\lfloor t_{k+1}\right\rfloor+1,\left\lfloor t_{k+2}\right\rfloor+1, \ldots,\left\lfloor t_{d}\right\rfloor+1\right)$. For any $T>0$, we define a Gaussian random field $\left\{Y_{T}(\mathbf{t}): \mathbf{t} \in[0, T]^{d}\right\}$ by

$$
Y_{T}(\mathbf{t}):=\left(1-\frac{R}{\log T}\right)^{1 / 2} \eta(\mathbf{t})+\left(\frac{R}{\log T}\right)^{1 / 2} \mathcal{W},
$$

where $\mathcal{W}$ denotes an $N(0,1)$ random variable independent of $\{\eta(\mathbf{t})\}$. Then the covariance $C_{T}(\mathbf{t}, \mathbf{t}+\mathbf{s}):=\operatorname{Cov}\left(Y_{T}(\mathbf{t}), Y_{T}(\mathbf{t}+\mathbf{s})\right)$ equals

$$
C_{T}(\mathbf{t}, \mathbf{t}+\mathbf{s})= \begin{cases}r(\mathbf{s})+(1-r(\mathbf{s})) \frac{R}{\log T} & \text { if }\left\lfloor s_{i}+t_{i}\right\rfloor=\left\lfloor t_{i}\right\rfloor \text { for } k<i \leqslant d, \\ \frac{R}{\log T} & \text { otherwise. }\end{cases}
$$

For $\mathbf{x} \in(0, \infty)^{d}$ we define $\mathbf{n}(\mathbf{x}, \mathbf{m}):=\left(n_{1}^{\mathbf{x}}, n_{2}^{\mathbf{x}}, \ldots, n_{d}^{\mathbf{x}}\right)$ with $n_{i}^{\mathbf{x}}:=x_{i} M_{i}$ for $i \in\{1,2, \ldots, k\}$ and $n_{i}^{\mathbf{x}}=n_{i}^{\mathbf{x}}(u):=\left\lfloor x_{i} m_{i}(u)\right\rfloor$ for $i \in\{k+1, k+2, \ldots, d\}$. Since

$$
\mathrm{P}\left(\sup _{\mathbf{t} \in \mathcal{R}_{\mathbf{m}}^{\mathrm{x}}} X(\mathbf{t}) \leqslant u\right)-\mathrm{P}\left(\sup _{\mathbf{t} \in \mathcal{R}^{\mathbf{n}(\mathbf{x}, \mathbf{m})}} X(\mathbf{t}) \leqslant u\right)=o(1), \quad \text { as } u \rightarrow \infty,
$$

we may focus on the asymptotics of the right-hand side of the above equality. 

boxes

S te $\mathrm{p} 1$. Let $\varepsilon>0$ be fixed. We divide the set $\mathcal{R}^{\mathbf{n}(\mathbf{x}, \mathbf{m})}$ into $n_{k+1}^{\mathbf{x}} n_{k+2}^{\mathbf{x}} \ldots n_{d}^{\mathbf{x}}$

$$
\mathcal{G}_{1}:=\prod_{i=1}^{k}\left[0, x_{i} M_{i}\right] \times \prod_{i=k+1}^{d}\left[l_{i}-1, l_{i}\right],
$$

indexed by $\mathbf{l}=\left(l_{k+1}, l_{k+2}, \ldots, l_{d}\right) \in \mathbb{N}^{d-k}$ such that $1 \leqslant l_{i} \leqslant n_{i}^{\mathbf{x}}$. Next we split each box $\mathcal{G}_{1}$ into two subsets $\mathcal{I}_{1}$ and $\mathcal{I}_{1}^{*}$ as follows:

$$
\begin{aligned}
\mathcal{I}_{\mathbf{l}} & :=\prod_{i=1}^{k}\left[0, x_{i} M_{i}\right] \times \prod_{i=k+1}^{d}\left[\left(l_{i}-1\right)+\varepsilon, l_{i}\right], \\
\mathcal{I}_{\mathbf{l}}^{*} & :=\mathcal{G}_{\mathbf{l}}-\mathcal{I}_{\mathbf{l}} .
\end{aligned}
$$

To simplify the notation, we will write

$$
\mathcal{I}:=\bigcup\left\{\mathcal{I}_{\mathbf{l}}: \mathbf{1} \leqslant \mathbf{1} \leqslant\left(n_{k+1}^{\mathbf{x}}, n_{k+2}^{\mathbf{x}}, \ldots, n_{d}^{\mathbf{x}}\right)\right\} .
$$

Applying the Bonferroni inequality, stationarity and the asymptotics (․․), we get

$$
\begin{aligned}
\limsup _{u \rightarrow \infty}\left|\mathrm{P}\left(\sup _{\mathbf{t} \in \mathcal{R}^{\mathbf{n}(\mathbf{x}, \mathbf{m})}} X(\mathbf{t}) \leqslant u\right)-\mathrm{P}\left(\sup _{\mathbf{t} \in \mathcal{I}} X(\mathbf{t}) \leqslant u\right)\right| \\
\quad \leqslant \limsup _{u \rightarrow \infty} n_{k+1}^{\mathbf{x}} n_{k+2}^{\mathbf{x}} \ldots n_{d}^{\mathbf{x}} \mathrm{P}\left(\sup _{\mathbf{t} \in \mathcal{I}_{\mathbf{1}}^{*}} X(\mathbf{t})>u\right) \leqslant \zeta_{1}(\varepsilon),
\end{aligned}
$$

uniformly for $\mathbf{x} \in[A, B]^{d}$, with $\zeta_{1}(\varepsilon) \rightarrow 0$ as $\varepsilon \rightarrow 0$.

S te p 2. Let $a>0$ be fixed and let $q_{1}, q_{2}, \ldots, q_{d}$ be defined as at the beginning of Section 3.3. Then we have

$$
\begin{aligned}
& \limsup _{u \rightarrow \infty}\left|\mathrm{P}\left(\sup _{\mathbf{t} \in \mathcal{I}} X(\mathbf{t}) \leqslant u\right)-\mathrm{P}\left(\sup _{\mathbf{j q} \in \mathcal{I}} X(\mathbf{j q}) \leqslant u\right)\right| \\
& \leqslant \limsup _{u \rightarrow \infty} n_{k+1}^{\mathbf{x}} n_{k+2}^{\mathbf{x}} \ldots n_{d}^{\mathbf{x}}\left|\mathrm{P}\left(\sup _{\mathbf{t} \in \mathcal{I}_{\mathbf{1}}} X(\mathbf{t}) \leqslant u\right)-\mathrm{P}\left(\sup _{\mathbf{j q} \in \mathcal{I}_{\mathbf{1}}} X(\mathbf{j q}) \leqslant u\right)\right| \\
& \leqslant \zeta_{2}(a),
\end{aligned}
$$

uniformly for $\mathbf{x} \in[A, B]^{d}$, with $\zeta_{2}(a) \rightarrow 0$ as $a \rightarrow 0$, due to the Bonferroni inequality and Lemma B.D.

$\mathrm{S}$ te $\mathrm{p}$ 3. Let $T$ be a function defined as follows:

$$
T(u):=B \max \left\{m_{1}(u), m_{2}(u), \ldots, m_{d}(u)\right\} .
$$

Note that if $T=T(u)$ is sufficiently large (and thus, if $u$ is sufficiently large), then

$$
\begin{aligned}
\left|r\left(\left(\mathbf{j}-\mathbf{j}^{\prime}\right) \mathbf{q}\right)-C_{T}\left(\mathbf{j} \mathbf{q}, \mathbf{j}^{\prime} \mathbf{q}\right)\right| & \leqslant \rho_{T}\left(\left(\mathbf{j}-\mathbf{j}^{\prime}\right) \mathbf{q}\right), \\
\left|C_{T}\left(\mathbf{j} \mathbf{q}, \mathbf{j}^{\prime} \mathbf{q}\right)\right| & \leqslant \varrho_{T}\left(\left(\mathbf{j}-\mathbf{j}^{\prime}\right) \mathbf{q}\right),
\end{aligned}
$$


where the functions $\rho_{T}$ and $\varrho_{T}$ are defined by (3.2). Moreover, for all pairs of points $\mathbf{j q}, \mathbf{j}^{\prime} \mathbf{q} \in \mathcal{I}$ satisfying $\left\|\mathbf{j}-\mathbf{j}^{\prime}\right\|_{\infty}<\varepsilon$, provided that $\varepsilon$ is sufficiently small, we obtain

$$
\begin{aligned}
& \left|r\left(\left(\mathbf{j}-\mathbf{j}^{\prime}\right) \mathbf{q}\right)-C_{T}\left(\mathbf{j} \mathbf{q}, \mathbf{j}^{\prime} \mathbf{q}\right)\right|=\frac{R \cdot\left(1-r\left(\left(\mathbf{j}-\mathbf{j}^{\prime}\right) \mathbf{q}\right)\right)}{\log T}, \\
& \max \left\{\left|r\left(\left(\mathbf{j}-\mathbf{j}^{\prime}\right) \mathbf{q}\right)\right|,\left|C_{T}\left(\mathbf{j} \mathbf{q}, \mathbf{j}^{\prime} \mathbf{q}\right)\right|\right\}=r\left(\left(\mathbf{j}-\mathbf{j}^{\prime}\right) \mathbf{q}\right)+\frac{R \cdot\left(1-r\left(\left(\mathbf{j}-\mathbf{j}^{\prime}\right) \mathbf{q}\right)\right)}{\log T} .
\end{aligned}
$$

Combining the above properties, the normal comparison lemma ([4], Theorem 4.2.1) and Lemmas 3.2 and 3.3 in the same way as in [3], we conclude that

$$
\lim _{u \rightarrow \infty}\left|\mathrm{P}\left(\sup _{\mathbf{j} \mathbf{q} \in \mathcal{I}} X(\mathbf{j q}) \leqslant u\right)-\mathrm{P}\left(\sup _{\mathbf{j} \mathbf{q} \in \mathcal{I}} Y_{T}(\mathbf{j q}) \leqslant u\right)\right|=0,
$$

uniformly for $\mathbf{x} \in[A, B]^{d}$.

S te p 4. By the definition of the random field $\left\{Y_{T}(\mathbf{t})\right\}$, we have

$$
\mathrm{P}\left(\sup _{\mathbf{j} \mathbf{q} \in \mathcal{I}} Y_{T}(\mathbf{j q}) \leqslant u\right)=\int_{-\infty}^{\infty} \mathrm{P}\left(\eta(\mathbf{j q}) \leqslant \frac{u-(R / \log T)^{1 / 2} z}{(1-R / \log T)^{1 / 2}} ; \mathbf{j q} \in \mathcal{I}\right) d \Phi(z) .
$$

Since $T=T(u)=\exp \left(\gamma u^{2}\right) c(u)$ for some function $c$ satisfying $\log c(u)=o\left(u^{2}\right)$, the condition

$$
\begin{aligned}
u_{z} & :=\frac{u-(R / \log T)^{1 / 2} z}{(1-R / \log T)^{1 / 2}} \\
& =\left(u-\left(\frac{R}{\log T}\right)^{1 / 2} z\right)\left(1+\frac{R}{2 \log T}+o\left(\frac{R}{\log T}\right)\right) \\
& =u+\frac{1}{u}\left(-\sqrt{\frac{R}{\gamma}} z+\frac{R}{2 \gamma}\right)+o\left(\frac{1}{u}\right)
\end{aligned}
$$

holds for every $z \in \mathbb{R}$. Moreover, as $u \rightarrow \infty$,

$$
\frac{m(u)}{m\left(u_{z}\right)}=\frac{u_{z}^{2 / \alpha_{1}} u_{z}^{2 / \alpha_{2}} \ldots u_{z}^{2 / \alpha_{d}} \Psi\left(u_{z}\right)}{u^{2 / \alpha_{1}} u^{2 / \alpha_{2}} \ldots u^{2 / \alpha_{d}} \Psi(u)} \rightarrow \exp \left(-\frac{R}{2 \gamma}+\sqrt{\frac{R}{\gamma} z}\right),
$$

and thus

$$
n_{k+1}^{\mathbf{x}} n_{k+2}^{\mathbf{x}} \ldots n_{d}^{\mathbf{x}}=\frac{x_{k+1} \ldots x_{d}}{M_{1} \ldots M_{k}} \exp \left(-\frac{R}{2 \gamma}+\sqrt{\frac{R}{\gamma} z}\right) m\left(u_{z}\right)(1+o(1)) .
$$

Applying the dependence structure of $\{\eta(\mathbf{t})\}$ and stationarity of $\{X(\mathbf{t})\}$, we obtain

$$
\mathrm{P}\left(\sup _{\mathbf{j} \mathbf{f} \in \mathcal{I}} \eta(\mathbf{j q}) \leqslant u_{z}\right)=\mathrm{P}\left(\sup _{\mathbf{j q} \in \mathcal{I}_{\mathbf{1}}} X(\mathbf{j q}) \leqslant u_{z}\right)^{n_{k+1}^{\mathbf{x}} n_{k+2}^{\mathbf{x}} \cdots n_{d}^{\mathbf{x}}}+o(1) .
$$


By Lemma B.1, the definition of $m\left(u_{z}\right)$ and properties (‥1) and (B.6), we get

$$
\begin{aligned}
& \mathrm{P}\left(\sup _{\mathbf{j} \mathbf{q} \in \mathcal{I}_{1}} X(\mathbf{j q}) \leqslant u_{z}\right)^{n_{k+1}^{\mathbf{x}} n_{k+2}^{\mathbf{x}} \ldots n_{d}^{\mathbf{x}}} \\
& \leqslant\left(\mathrm{P}\left(\sup _{\mathbf{t} \in \mathcal{G}_{1}} X(\mathbf{t}) \leqslant u_{z}\right)+\frac{\prod_{i=1}^{k} M_{i} x_{i} \cdot(\vartheta(a)+2 \varepsilon+o(1))}{m\left(u_{z}\right)}\right)^{n_{k+1}^{\mathbf{x}} n_{k+2}^{\mathbf{x}} \cdots n_{d}^{\mathbf{x}}} \\
&=\left(1-\frac{\prod_{i=1}^{k} M_{i} x_{i} \cdot(1-\vartheta(a)-2 \varepsilon+o(1))}{m\left(u_{z}\right)}\right)^{\frac{x_{k+1} \ldots x_{d}}{M_{1} \ldots M_{k}} \exp \left(-\frac{R}{2 \gamma}+\sqrt{\frac{R}{\gamma}} z\right) m\left(u_{z}\right)}+o(1) \\
& \underset{u \rightarrow \infty}{\longrightarrow} \exp \left(-(1-\vartheta(a)-2 \varepsilon) x_{1} x_{2} \ldots x_{d} \exp \left(-\frac{R}{2 \gamma}+\sqrt{\frac{R}{\gamma}} z\right)\right)
\end{aligned}
$$

where $\vartheta(a) \rightarrow 0$ as $a \rightarrow 0$. Thus

$$
\begin{aligned}
& \limsup _{u \rightarrow \infty} \int_{-\infty}^{\infty} \mathrm{P}\left(\sup _{\mathbf{j} \mathbf{q} \in \mathcal{I}_{1}} X(\mathbf{j q}) \leqslant u_{z}\right)^{n_{k+1}^{\mathbf{x}} n_{k+2}^{\mathbf{x}} \ldots n_{d}^{\mathbf{x}}} d \Phi(z) \\
& \quad \leqslant \operatorname{E} \exp \left(-(1-\vartheta(a)-2 \varepsilon) x_{1} x_{2} \ldots x_{d} \exp \left(-\frac{R}{2 \gamma}+\sqrt{\frac{R}{\gamma}} \mathcal{W}\right)\right) .
\end{aligned}
$$

On the other hand, we have

$$
\begin{aligned}
& \mathrm{P}\left(\sup _{\mathbf{j q} \in \mathcal{I}_{\mathbf{1}}} X(\mathbf{j q}) \leqslant u_{z}\right)^{n_{k+1}^{\mathbf{x}} n_{k+2}^{\mathbf{x}} \ldots n_{d}^{\mathbf{x}}} \\
& \geqslant \mathrm{P}\left(\sup _{\mathbf{t} \in \mathcal{G}_{1}} X(\mathbf{t}) \leqslant u_{z}\right)^{n_{k+1}^{\mathbf{x}} n_{k+2}^{\mathrm{x}} \cdots n_{d}^{\mathbf{x}}} \\
& \geqslant\left(1-\frac{\prod_{i=1}^{k} M_{i} x_{i}}{m\left(u_{z}\right)}\right)^{\frac{x_{k+1} x_{k+2} \ldots x_{d}}{M_{1} M_{2} \ldots M_{k}} \exp \left(-\frac{R}{2 \gamma}+\sqrt{\frac{R}{\gamma}} z\right) m\left(u_{z}\right)}+o(1) \\
& \underset{u \rightarrow \infty}{\longrightarrow} \exp \left(-x_{1} x_{2} \ldots x_{d} \exp \left(-\frac{R}{2 \gamma}+\sqrt{\frac{R}{\gamma} z}\right)\right) \text {, }
\end{aligned}
$$

and thus

$$
\begin{aligned}
\liminf _{u \rightarrow \infty} \int_{-\infty}^{\infty} \mathrm{P}\left(\sup _{\mathbf{j q} \in \mathcal{I}_{\mathbf{1}}}\right. & \left.X(\mathbf{j q}) \leqslant u_{z}\right)^{n_{k+1}^{\mathbf{x}} n_{k+2}^{\mathbf{x}} \ldots n_{d}^{\mathbf{x}}} d \Phi(z) \\
\geqslant & \operatorname{E} \exp \left(-x_{1} x_{2} \ldots x_{d} \exp \left(-\frac{R}{2 \gamma}+\sqrt{\frac{R}{\gamma}} \mathcal{W}\right)\right) .
\end{aligned}
$$


Summarizing, we obtain

$$
\begin{aligned}
& \operatorname{E} \exp \left(-x_{1} x_{2} \ldots x_{d} \exp \left(-\frac{R}{2 \gamma}+\sqrt{\frac{R}{\gamma}} \mathcal{W}\right)\right) \\
& \quad \leqslant \liminf _{u \rightarrow \infty} \mathrm{P}\left(\sup _{\mathbf{j} \mathbf{i} \in \mathcal{I}} Y_{T}(\mathbf{j q}) \leqslant u\right) \leqslant \limsup _{u \rightarrow \infty} \mathrm{P}\left(\sup _{\mathbf{j} \mathbf{q} \in \mathcal{I}} Y_{T}(\mathbf{j q}) \leqslant u\right) \\
& \quad \leqslant \operatorname{E} \exp \left(-(1-\vartheta(a)-2 \varepsilon) x_{1} x_{2} \ldots x_{d} \exp \left(-\frac{R}{2 \gamma}+\sqrt{\frac{R}{\gamma} \mathcal{W}}\right)\right),
\end{aligned}
$$

uniformly for $\mathbf{x} \in[A, B]^{d}$.

$\mathrm{S}$ te $\mathrm{p}$ 5. From Steps $1-3$ of the proof we know that

$$
\limsup _{u \rightarrow \infty}\left|\mathrm{P}\left(\sup _{\mathbf{t} \in \mathcal{R}^{\mathbf{n}(\mathbf{x}, \mathbf{m})}} X(\mathbf{t}) \leqslant u\right)-\mathrm{P}\left(\sup _{\mathbf{j q} \in \mathcal{I}} Y_{T}(\mathbf{j q}) \leqslant u\right)\right| \leqslant \zeta_{1}(\varepsilon)+\zeta_{2}(a),
$$

uniformly for $\mathrm{x} \in[A, B]^{d}$, with $\zeta_{1}(\varepsilon) \rightarrow 0$ as $\varepsilon \rightarrow 0$ and $\zeta_{2}(a) \rightarrow 0$ as $a \rightarrow 0$. Combining it with the inequalities (3.7) and passing with $\varepsilon \rightarrow 0$ and $a \rightarrow 0$, we finish the first part of the proof.

(ii) Let $\mathcal{J} \subset \mathbb{R}^{d}$ be an arbitrary Jordan-measurable set with Lebesgue measure $\lambda(\mathcal{J})>0$. We follow the argumentation from [3], Theorem 2 (ii). Observe that for every $\varepsilon>0$ there exist some positive constants $z_{1}, z_{2}, \ldots, z_{d}$ and some sets $\mathcal{L}_{\varepsilon}, \mathcal{U}_{\varepsilon} \subset \mathbb{R}^{d}$ being finite sums of disjoint closed hyperrectangles with dimensions $z_{1} \times z_{2} \times \ldots \times z_{d}$, such that $\mathcal{L}_{\varepsilon} \subset \mathcal{J} \subset \mathcal{U}_{\varepsilon}$ and $\lambda\left(\mathcal{L}_{\varepsilon}\right)+\varepsilon>\lambda(\mathcal{J})>\lambda\left(\mathcal{U}_{\varepsilon}\right)-\varepsilon$. Then, following nearly line-by-line the arguments given in the proof of part (i), we obtain

$$
\mathrm{P}\left(\sup _{\mathbf{t} \in\left(\mathcal{L}_{\varepsilon}\right)_{\mathbf{m}}^{\mathbf{x}}} X(\mathbf{t}) \leqslant u\right) \rightarrow \operatorname{E} \exp \left(-x_{1} x_{2} \ldots x_{d} \lambda\left(\mathcal{L}_{\varepsilon}\right) \exp \left(-\frac{R}{2 \gamma}+\sqrt{\frac{R}{\gamma}} \mathcal{W}\right)\right)
$$

and

$$
\mathrm{P}\left(\sup _{\mathbf{t} \in\left(\mathcal{U}_{\varepsilon}\right)_{\mathbf{m}}^{\mathbf{x}}} X(\mathbf{t}) \leqslant u\right) \rightarrow \mathrm{E} \exp \left(-x_{1} x_{2} \ldots x_{d} \lambda\left(\mathcal{U}_{\varepsilon}\right) \exp \left(-\frac{R}{2 \gamma}+\sqrt{\frac{R}{\gamma}} \mathcal{W}\right)\right),
$$

as $u \rightarrow \infty$, uniformly for $\mathbf{x} \in[A, B]^{d}$. Since $\varepsilon>0$ is arbitrarily small, it gives

$$
\mathrm{P}\left(\sup _{\mathbf{t} \in \mathcal{J}_{\mathbf{m}}^{\mathbf{x}}} X(\mathbf{t}) \leqslant u\right) \rightarrow \mathrm{E} \exp \left(-x_{1} x_{2} \ldots x_{d} \lambda(\mathcal{J}) \exp \left(-\frac{R}{2 \gamma}+\sqrt{\frac{R}{\gamma}} \mathcal{W}\right)\right),
$$

as $u \rightarrow \infty$, uniformly for $\mathbf{x} \in[A, B]^{d}$, which completes the proof.

Acknowledgments. The author would like to thank Krzysztof Dębicki and Michał Kukieła for comments and suggestions. 


\section{REFERENCES}

[1] M. Arendarczyk and K. Dębicki, Exact asymptotics of supremum of a stationary Gaussian process over a random interval, Statist. Probab. Lett. 82 (2012), pp. 645-652.

[2] K. Dębicki, E. Hashorva, and N. Soja-Kukieła, Extremes of stationary Gaussian random fields, preprint, available at http://arxiv.org/abs/1312.2863, 2013.

[3] K. Dębicki, E. Hashorva, and N. Soja-Kukieła, Extremes of stationary Gaussian random fields, J. Appl. Probab. 52 (2015), pp. 55-67.

[4] M. R. Leadbetter, G. Lindgren, and H. Rootzén, Extremes and Related Properties of Random Sequences and Processes, Springer Ser. Statist., Springer, New York 1983.

[5] J. III Pickands, Asymptotic properties of maximum in a stationary Gaussian process, Trans. Amer. Math. Soc. 145 (1969), pp. 75-86.

[6] V. I. Piterbarg, Asymptotic Methods in the Theory of Gaussian Processes and Fields, Transl. Math. Monogr., Vol. 148, American Mathematical Society, Providence 1996.

[7] Z. Tan and E. Hashorva, Exact tail asymptotics of the supremum of a strongly dependent Gaussian process over a random interval, Lith. Math. J. 53 (2013), pp. 91-102.

Natalia Soja-Kukieła

Faculty of Mathematics and Computer Science

Nicolaus Copernicus University

ul. Chopina 12/18

87-100 Toruń, Poland

E-mail: natas@mat.umk.pl 